\title{
On Heartbreak in Oncology
}

Daniel Rayson ${ }^{1}$

Published online: 25 January 2019

(C) Springer Science+Business Media, LLC, part of Springer Nature 2019

Keywords Burnout $\cdot$ Stress $\cdot$ Coping $\cdot$ Heartbreak

The many successes cancer medicine has witnessed over the past 2 decades have dramatically escalated the burden of care that oncologists are expected to provide. The availability of multiple treatment lines for diseases previously approached with therapeutic nihilism coupled with significant improvements in outcomes has been a boon to all those affected by cancer. These same achievements have also dramatically increased patient and societal expectations and have impacted the burden of care while blurring the lines between previously stark transitions from active to palliative or hospice care beyond recognition. The prolongation of active treatment times for almost all disease subtypes has resulted in a significant increase in the number of clinical stress points within an oncologist-patient relationship.

There has been much written regarding optimal communication and coping strategies for patients moving from active oncologic care to full palliative care or hospice. Many resources have been brought to bear to help both clinicians and patients navigate this period in the disease course that comes for so many. In some ways, this single transition has become the prototypical stress point of maximal heartache and existential angst, the one we see in movies and read about in novels. It is a part of the collective oncologic and societal atmosphere.

Without minimizing the importance of this transition, the cumulative impact of many other stress points, with most flying under the radar for those not in the clinic room, may be more

This article is part of the Topical Collection on Palliative Medicine

Section Editor: A Jatoi

Daniel Rayson

daniel.rayson@nshealth.ca

1 Division of Medical Oncology, Queen Elizabeth II Health Sciences Centre and Dalhousie University, Room 457A Bethune Building, 1276 South Park St., B3H2Y9, Halifax, Nova Scotia, Canada treacherous to manage. The sequential and additive weight of these stressors coupled with longer survival times can increase their "area under the curve" to potentially toxic levels.

The 41-year-old mother of 2 with a newly diagnosed breast cancer and an incidentally discovered 6-mm pulmonary nodule who is convinced beyond all reason that she has metastatic disease and with whom you plead to consider adjuvant chemotherapy despite her certainty that it will be futile. The 53year-old man trying to continue to work as a painter despite weekly chemotherapy sessions for his metastatic colon cancer. He does not get paid if he does not work. His wife and children urged him on, "we'll manage Dad-you have to do this." The 26-year-old with a metastatic sarcoma making a decision to proceed to his 3 rd pulmonary resection with parents desperately searching for the next step in his care and unable to afford travel for clinical trial participation. Exhausted beyond comprehension they are holding together only through the shared love of him, their only child, their life for the past 26 years.

None of these scenarios are unique and we all play a part in their evolution. On a typical clinic day, multiple iterations play out. They all have their own plot twist and we never know what lies behind the closed examination room door until we open it to reveal the next dilemma, the next decision, the next tragedy.

It is easy to become complacent in regard to the psychological and emotional impact of these types of clinical encounters and dilemmas. New technology, additional lines of therapy, and improving survival expectations for experimental arms in clinical trials dominate the news and the plenary sessions at international meetings. These advances insert themselves into our onco-consciousness as we approach each patient with the latest treatment option with a lighter step and a little more hope. Both oncologist and patient grab on to this hope but the path is never simple and even in the best scenarios, with optimal outcomes, there are multiple episodes of uncertainty, trauma, and pain that accompany clinic visits. 
In most environments, we are on our own with little professional support to help us cope with the emotional toll of repetitive exposure to cancer narratives. Our footprints are in the systemic therapy orders we write and the clinic letters we dictate that focus on the clinical symptoms, tolerance of therapy, lab work, investigations, and treatment plan. The stuff that matters.

There is, however, little evidence that a casual observer can glean from these clinical droppings related to the emotional state of the oncologist doing the ordering and dictating. What was her day like? How did she feel giving this patient the bad news? How did she discuss the new lung metastases? How did she feel looking into the eyes of this patient whom she has known for 4 years and telling her that there are no reasonable options left? What was she thinking about on the drive home? How did she greet her partner? What did she make for supper for the kids? Did she fall asleep with her daughter holding her tight? How will she do it tomorrow, and the day after, and the day after?

With all due respect to our colleagues in palliative medicine, and with the acknowledgment of recent data supporting the earlier integration of palliative care alongside active noncurative cancer treatment, once the end is near and is clear for all to see with both patients and their loved ones having accepted symptomatic and supportive care alone, helping patients die with comfort and dignity can be relatively straightforward. We have the medications, the care teams, and the structures to make this happen. We have all witnessed the peace and acceptance that can come once the bloodwork and scans stop, the lines of therapy come to an end, the cumulative treatment toxicities abate, and the waiting for the next bad thing to happen ends once there is acceptance of the ultimate bad thing, inevitable death.

It is the time between day 1 of active oncology care and day 1 of fully transitioned supportive care that, in large part, defines the essence of an oncologists' career choice and represents the significant value we bring to the table as clinicians and as individuals. The way we manage this time in our own heads and lives influences our professional resilience and psychodynamic stability and often that of our loved ones as well. The way we remember these times over the course of our career from an individual and collective perspective defines our narrative, our story, and our reason for having chosen a career in oncology in the first place.

It may be easier and protective not to think about all this and to bury the clinical challenges under layers of data, endpoints, and $p$-values. Some degree of separation between us and the daily clinical and existential pain our patients face may, in fact, be critical. This separation takes both conscious and subconscious work to maintain, however and is at risk of being breached at any time. Over the long term and applied too broadly, it is a strategy that can negatively impact communication style and supportive care expertise. Taken to extremes, it can define a reputation and career in ways not otherwise intended. The work it takes to maintain this separation can bleed into other areas impacting personal relationships, family, and friends. The breaches can be insidious as well as cumulative and, like most poorly constructed edifices, structural failures have secondary impacts which typically come at great cost.

Most who wonder how we do the jobs we do are not asking the question because of the inordinate complexity of cancer care, the exploding treatment options that we have at our disposition, or the fascinating science underlying the mechanisms of malignant disease that seem to be revealing themselves on a monthly basis. They ask that question because they don't know how we stay sane repeatedly dealing with lifethreatening illness, cancer-related anxiety, and the existential crises our patients continuously face.

Over the course of a typical year in the life of an oncologist or oncology nurse, there are many stories that can be brought home to disturb sleep and disrupt relationships. They can intrude when one least expects and continue to haunt long after the initial experience has passed. The hundreds of stories that make up the professional life of those working in cancer medicine put us all at risk for a cousin to post-traumatic stress disorder (PTSD) termed continuous traumatic stress disorder or CTSD. Initially conceived as a reaction to repetitive trauma in situations of societal inequality and deprivation, familial dysfunction, or violence and life in war zones, CTSD seems to be an apt description for the continuous exposure to situations that force us to revisit painful experiences as we take care of cancer patients and their families [1].

Moving between clinic rooms on a typical day and trying to instill the right mix of hope, comfort, and guidance within the context of setting realistic expectations while providing continuously evolving state of the art medical and supportive care is quietly exhausting. Under the radar, most of us have been "white knuckling" at some point in our careers and many will experience elements of CTSD that go unrecognized by our colleagues, friends, families, or ourselves [2]. In the end, resilience and duty can take a toll on the unsuspecting and vulnerable.

The lines between reactive depression, compassion fatigue, career burnout, and CTSD seem especially blurred in our field due to the plethora of clinical, institutional, and existential stresses faced by all, and the intense and continuous energy that is required to maintain mental health and emotional stability [3] References abound in relation to these constructs regarding their incidence, risk mitigation, and overall importance to the oncology workforce. Most of these references approach issues in starkly clinical terms that do not always capture the essence of the emotional component underlying the stresses we face on a continuous basis $[4,5]$. 
For most of us, the term heartbreak has no direct medical connotation and is a concept relegated to music, literature, or high school relationships. The concept has similar meanings in many cultures and is hypothesized to relate to a universal adaptive mechanism designed to encourage the maintenance of social relationships. The essence is that it is a somatic and psychological state that leads one to work harder to maintain relationships in order to avoid the pain that comes with heartbreak [6]. One way to avoid heartbreak is to never let our hearts into the equation but that is a treacherous and likely unsustainable strategy for most. I would suggest that we accept and embrace the concept as an apt description of a component of what it means to be a medical oncologist.

The heartbreak in oncology is not limited to a solitary event in time such as with transition to palliative care or hospice. There are multiple points within the continuum of care where heartbreak rises up. The progressing scan, the new symptom that we cannot seem to fully control, the intolerably toxic treatment that we recommended and that ended up doing more harm than good, the existential and questions we try to answer in 30-s sound bites due to the pressures of a full waiting room. All of these phenomena arise in a dose-dense fashion unique to oncology and in the context of an unbalanced relationship where the oncologist is seen as having the answers at all times, in all situations, and for all comers.

Heartbreak is perhaps most acute when we take care of young patients with lives interrupted. Although the median age at cancer diagnosis is 66 and fewer than $10 \%$ of cancers arise in persons below the age of 45 , these are the situations that tend to resonate most acutely, particularly when favorable outcomes are expected but do not happen [6]. The unfairness of a cancer diagnosis in a young person, or death from testicular cancer, or node-negative breast cancer when all guidelines and evidence suggest that death should not happen can disrupt any coping and resilience built up through training and experience triggering; let us call it what it is, heartbreak.

We need to move away from the sterile descriptions of both clinical medicine and psychology to truly grasp the nature of the experience. Some will find solace, understanding, and strength in music, literature, philosophy, or art. Others will need to exercise their way through the pain. Still, others will find the same comfort or release with family, friends, and other relationships or through the collective support and shared experience of other colleagues dropping into the office when hearing about a particularly difficult case. Some will benefit from active listening, others from guidance and discussion of coping of strategies, and still others will just need a knowing look or hand on the shoulder saying "I have been there. I know. And I'm here if you need me."

We cannot keep rushing through the heartbreak. Time needs to be taken to recognize, acknowledge, feel, and reconstitute. Each one of us will have a different way of doing this and will need support to be able to do so in unique and individualized ways, both formal and informal. Even the most senior of us will need help periodically, but perhaps the most important way forward is to make sure that trainees and junior staff have the tools and space to develop the strategies that will be most helpful to them over the course of their career.

Failure to recognize heartbreak in oncology comes with a cost. Burnout, alcohol abuse, illicit drugs, abusive relationships, bad clinical behavior, fractured families, self-harm, and suicide are all potential consequences of failing to recognize the intense emotional work of what we do. There is a reason heartbreak is such a common theme in music and literature. It speaks to an essential component of our humanity. The capacity to attach, to care, and to protect is wired in our DNA. The pain that comes with failure in any of these domains is essentially universal.

A typical relationship has a number of points of tension but the relationship between oncologist and patient takes things to a different level. The imbalance in power and knowledge coupled with persistent patient desperation and physical as well as an existential threat makes attachment e specially treacherous, particularly as the clinical situation deteriorates.

Our hearts break a little every week and resiliency comes with the development of adaptations that lead to remodelling and repair. There is no one fix for all nor is there a single pathway to help us navigate. We need to look beyond traditional psychological or medical concepts to others that may resonate more closely with many of us in clinical oncology. The heartbreak that comes with our jobs is both a gift and a threat. We need to call it what it is in order to move past the pain, just like the songs say.

\section{Compliance with Ethics Guidelines}

Conflict of Interest Daniel Rayson declares that he has no conflict of interest.

Human and Animal Rights and Informed Consent This article does not contain any studies with human or animal subjects performed by any of the authors.

Publisher's Note Springer Nature remains neutral with regard to jurisdictional claims in published maps and institutional affiliations.

\section{References}

1. Eagle G, Kaminer D. Continuous traumatic stress: expanding the lexicon of traumatic stress. Peace and Conflict: Journal of Peace Psychology. 2013;19(2):85-99.

2. Rayson D. White Knuckling. J Clin Oncol. 2016;34(12):1419-20. 
3. Gieseler F, Gaertner L, Thaden E, Theobald W. Cancer diagnosis: a trauma for patients and doctors alike. Oncologist. 2018;23:752-4.

4. West CP, Dyrbye LN, Erwin PJ, Shanafelt TD. Interventions to prevent and reduce physician burnout: a systematic review and metaanalysis. Lancet. 2016;388:2272-81.
5. Wikipedia contributors. Broken Heart; https://en.wikipedia.org/w/ index.php?title=Broken heart\&oldid=855544007, accessed Aug 27 2018.

6. Age and cancer risk; https://www.cancer.gov/aboutcancer/causesprevention/risk/age, accessed Jan 142019. 